\title{
ESTIMACIÓN DEL RIESGO SÍSMICO EN CASTILLA - LA MANCHA PARA LA ELABORACIÓN DEL PLAN ESPECIAL DE EMERGENCIAS
}

\author{
AN ESTIMATION OF THE SEISMIC RISK IN CASTILLA - LA MANCHA IN ORDER TO DEVELOP THE SPECIAL \\ EMERGENCY PROGRAMME
}

\author{
Rocío Romero-Jarén “, Ligia Quirós, Fernando Sorondo, Belén Benito
}

Dpto. Ingeniería Topográfica y Cartografia, Universiad Politécnica de Madrid, Crta de Valencia km 7, 28031, Madrid, España. rocio.rjaren@alumnos.upm.es; l.quiros@alumnos.upm.es

\begin{abstract}
:
An estimation of the seismic risk in Castilla - La Mancha (Spain) is set forth in the current work, in order to develop the special emergency programme. To carry out the study it has been necessary to define a multidisciplinary group of experts in each involved discipline: geology and tectonics, seismology, architecture, engineering and geographical information systems. The main aim is to develop different seismic risk maps to provide the basis to elaborate the emergency plans in Castilla - La Mancha. These plans must follow the stipulated guidelines in the seismic risk field. A probabilistic methodology is adopted to define the seismic risk, considering this as the human and material losses in presence of the expected seismic event. The seismic hazard of the area of study is evaluated through return periods of 475 and 975 years, equivalents to exceedance probabilities of $10 \%$ and $5 \%$ in 50 years respectively. These probabilities are proposed in the framework of the Spanish seismic code "Normativa Sismorresistente Española, NCSE-02", for conventional and special buildings. In a first approach, the study attempt to estimates the expected losses in each city of the overall of Castilla - La Mancha in the presence of the probable movements in 50 and 100 years. The results allow us to make a relative estimation of the seismic risk in different areas, identify those cities which undergo highest damages indexes and which ones would require a more in-depth assessment so as to mitigate the risk. Besides, the results contribute to establish objective priorities to define emergency plans at city scale.
\end{abstract}

Key words: Geographical Information System, Geophysics, Seismic Hazard, Seismic Risk, Geology

\section{Resumen:}

El estudio que se presenta a continuación cosiste en la estimación del riesgo sísmico en Castilla - La Mancha (España) para determinar el grado de péridas esperadas ante eventuales terremotos futuros. Este trabajo ha sido realizado por un grupo multidisciplinar, compuesto por especialistas en todas las disciplinas involucradas: geología y tectónica, sismología, arquitectura, ingeniería y sistemas de información geográfica. El objetivo global del estudio es la elaboración de mapas de riesgo sísmico que sirvan de base para la elaboración de planes de emergencia en Castilla - La mancha, siguiendo la directriz básica que Protección Civil tiene encomendada en materia de riesgo sísmico. El riesgo se define siguiendo un planteamiento probabilista, como el grado de pérdidas humanas y materiales ante la acción sísmica esperada en cada punto del territorio con una determinada probabilidad de excedencia o periodo de retorno. En concreto, el estudio se lleva a cabo para periodos de retorno de 475 y 975 años, que equivale a la probabilidad de excedencia del $10 \%$ en 50 años y $5 \%$ en 50 años respectivamente. Estas son las probabilidades adoptadas en la Normativa Sismorresistente Española NCSE-02 para regular el diseño de estructuras de edificación convencionales y de especial importancia. Se ha tratado así de estimar, en una primera aproximación, el grado de pérdidas que cabe esperar en cada término municipal ante los movimientos con las probabilidades mencionadas en toda la Comunidad de Castilla La Mancha. Los resultados permiten establecer una valoración relativa del riesgo en las diferentes zonas e identificar aquellos municipios que, por su mayor índice de riesgo, requieran estudios de detalle para proceder a su mitigación. Por otro lado, los resultados aportan un criterio objetivo para establecer prioridades en la definición de planes de emergencia a escala municipal.

Palabras clave: Sistema de información geográfica, Geofísica, Peligrosidad sísmica, Riesgo sísmico, Geología

\section{Introducción}

El presente estudio de riesgo sísmico se ha desarrollado para la Comunidad Autónoma de Castilla - La Mancha con el objetivo global obtener mapas de distintos parámetros indicativos del riesgo al que está expuesto cada termino municipal, que sirvan de base para la elaboración del plan de emergencias de dicha Comunidad Autónoma por parte de Protección Civil.

Según la definición de UNDRO (1979) oficialmente aceptada, el riesgo sísmico, $R$, en una determinada población, se define tal y como se muestra en la Ec. (1).

$R=H \cdot V \cdot E \cdot C$

Donde: 
$\mathrm{H}$ es la peligrosidad sísmica que determina el movimiento esperado en la población.

V es la vulnerabilidad de las estructuras. habitantes.

E es la exposición o densidad de estructuras y

C es el coste de reparación o de pérdidas.

Siguiendo estrictamente la definición anterior, el riesgo vendría expresado en términos económicos, que representarían los costes de reparación o reconstrucción por pérdidas ante el movimiento estimado en el cálculo de la peligrosidad. Numerosas variantes han sido propuestas, a fin de expresar el riesgo en otros términos, por ejemplo, porcentaje de un determinado grado de daño en una cierta tipología estructural, daño medio, número de víctimas mortales y heridos, número de viviendas inhabitables, etc.

La elección del índice o parámetro de riesgo depende de la aplicación a la que vaya dirigido el estudio, pero en cualquier caso su estimación requiere conocer: 1) la peligrosidad o amenaza sísmica a la que está expuesta la población, 2) la distribución de vulnerabilidad sobre el parque inmobiliario en la zona, es decir, el número de estructuras de cada tipología o clase de vulnerabilidad, y 3) la relación entre el movimiento de entrada y los daños esperados en cada tipología, es decir, las curvas de fragilidad. Esta es la secuencia de actuación que se sigue en el presente estudio de riesgo, en el que se obtienen, como principales resultados, los daños físicos en las edificaciones (ligero, moderado, extenso, completo), las pérdidas humanas y las pérdidas económicas para los escenarios sísmicos definidos.

Se comienza estimando la amenaza y caracterizando la acción sísmica que sirve como input para el posterior cálculo del riesgo. Se caracterizan así los movimientos esperados por sismos futuros, con dos probabilidades de excedencia: $10 \%$ en 50 años y $5 \%$ en 50 años, movimientos correspondientes a periodos de retorno PR 475 y PR 975 años, respectivamente. Estas probabilidades son las adoptadas por la Normativa Sismorresistente Española, NCSE-02, para regular el diseño de estructuras de edificaciones convencionales (PR 475) y de especial importancia (PR 975)

Para poder realizar las estimaciones de peligrosidad y riesgo sísmico, es necesario construir una base de datos georreferenciada (BD) que, a través de un Sistema de Información Geográfica, permita el almacenamiento, edición, análisis y representación de la información compilada.

El estudio se ha articulado en cinco fases principales (Fig. 1), que se han desarrollado paralela o sucesivamente con el fin de cubrir todos los aspectos que requiere un estudio de riesgo sísmico:

1. Elaboración de la base de datos con toda la información sobre la sismicidad, tectónica, exposición de las viviendas y la población, división administrativa, etc.

2. Evaluación y cálculo de la peligrosidad sísmica.

3. Análisis de la exposición y asignación de vulnerabilidad.
4. Estimación del riesgo sísmico.

5. Creación de un SIG con los resultados de riesgo sísmico.

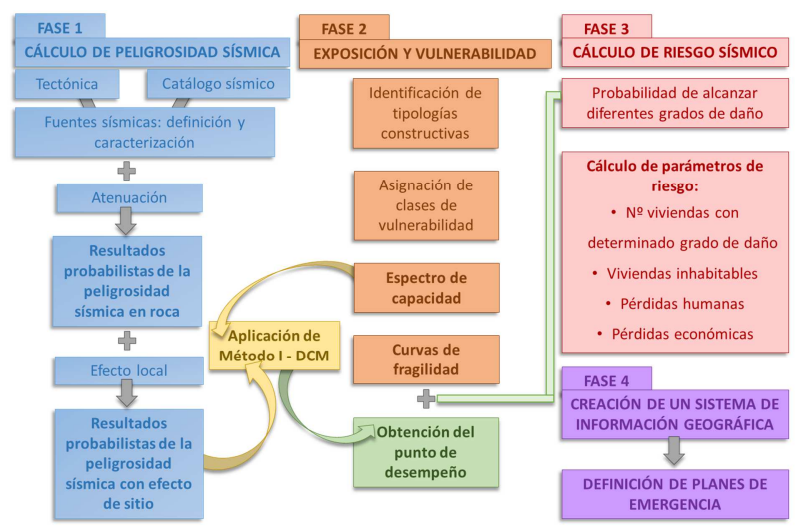

Figura 1: Organigrama de trabajo con las diferentes fases del estudio (fuente: elaboración propia).

\section{Peligrosidad sísmica}

La estimación de la peligrosidad sísmica se efectúa teniendo en cuenta todas las fuentes incluidas en un área de influencia de $300 \mathrm{~km}$ alrededor del centroide de Castilla - La Mancha. La extensión del área de influencia es la que habitualmente se fija en las normativas.

\subsection{Metodología probabilista}

La concepción probabilista de la peligrosidad sísmica considera la probabilidad de que se iguale o supere un determinado nivel de movimiento del terreno, como resultado de la acción de terremotos en el área estudio durante un periodo de tiempo especificado.

La Ec. (2) proporciona la peligrosidad en un emplazamiento por una única fuente, dada como probabilidad anual de excedencia de un nivel del movimiento $Y$ del parámetro $y$.

$$
\begin{gathered}
P_{\text {anual }}(y>Y)=\lambda_{\text {anual }}(y>Y)= \\
=\tau \iiint P(y>Y \mid m, r, \varepsilon) \cdot p(m) \cdot p(r) \cdot p(\varepsilon) d m d r d \varepsilon
\end{gathered}
$$

Donde:

$y$ representa el parámetro de movimiento con el que expresamos la peligrosidad (habitualmente aceleración pico PGA, o aceleración espectral SA (T)).

$Y$ es el valor fijado de movimiento cuya excedencia se evalúa.

$\tau$ es la tasa anual de ocurrencia de sismos y se considera constante dentro de una misma fuente.

$p(m)$ se refiere a la función de densidad de probabilidad de la magnitud dentro de cada fuente. Describe la probabilidad de que ocurra un terremoto de una determinada magnitud en la fuente sísmica y representa directamente el denominado efecto de la fuente.

$p(r)$ se refiere a la función de densidad de probabilidad de la distancia. Describe la probabilidad de que si se produce un terremoto en la fuente, su foco se encuentre a una distancia $r$ del emplazamiento de cálculo. Esta función representa la posible localización 
de un sismo dentro de la fuente sísmica y se relaciona con el efecto de la atenuación del movimiento a lo largo del trayecto entre la fuente y el emplazamiento.

$p(\varepsilon)$ está relacionada con la función de densidad de probabilidad asociada a la incertidumbre del movimiento que puede registrarse en el emplazamiento debido a un sismo determinado. Así, dadas una magnitud $\mathrm{m}$ y una distancia $\mathrm{r}$, cabe esperar distintos niveles de movimiento dada la aleatoriedad del mismo, que se considera mediante una distribución log normal de y en base a observaciones empíricas. El parámetro $\varepsilon$ indica precisamente el número de desviaciones estándar que se consideran para estimar y.

$P(y>Y \mid m, r, \varepsilon)$ es un término de probabilidad que refleja si se produce o no excedencia del nivel de movimiento prefijado al calcular el movimiento esperado para unas variables $m, r$ y $\varepsilon$ determinadas. Es por tanto un término que toma dos valores: 1 cuando sí se produce excedencia y 0 en caso contrario.

La integral triple se extiende al rango de variación de las tres variables $(m, r$ y $\varepsilon$ ) y se resuelve numéricamente, dado que en la mayor parte de los casos no existe solución analítica. Sumando la contribución de todas las zonas se obtiene la tasa anual de excedencia del movimiento o peligrosidad sísmica expresada en términos anuales.

Bajo la hipótesis de que la excedencia del movimiento es un proceso poissoniano, entonces esta probabilidad anual se traduce en una probabilidad en $t$ años mediante la Ec. (3)

$P(y>Y$ en $t$ años $)=1-e^{-t \cdot \lambda_{\text {anual }}}$

En concreto, en el presente estudio de peligrosidad se emplean dos métodos de cálculo, ambos de tipo probabilista:

- Método Clásico Zonificado (MCZ), en el que se considera que la sismicidad se distribuye en zonas sismogenéticas, cada una de las cuales tiene un potencial sísmico uniforme (Cornell 1968; Esteva 1968)

- Método Híbrido de Zonas y Fallas (MHZF), en el que las fallas activas conocidas son modelizadas como fuentes independientes con su potencial sísmico asociado derivado de la tasa de sismicidad de las fallas y el resto de la sismicidad se reparte en zonas sismogenéticas (Rivas 2014).

El cálculo de peligrosidad sísmica engloba distintas fases. Éstas se explican a continuación.

\subsection{Fases del estudio de peligrosidad sísmica}

\subsubsection{Análisis de sismicidad, tectónica y geodinámica de la zona}

El acercamiento entre Iberia y África es el motor principal de la deformación activa que se observa en Castilla - La Mancha, denominada deformación Bética. En el interior de la Península se registra un incremento progresivo de los esfuerzos tectónicos con el máximo acortamiento horizontal en sentido NO-SE (Capote et al. 1990). A este incremento se asocia la deformación Intraplaca. El inicio de la transmisión de esfuerzos desde el SE coincide con el final de la actividad orogénica Pirenaica (Muñoz Martín 1997). En el interior peninsular, la transmisión de esfuerzos de origen Bético produce la reorganización del Antepaís Ibérico centrooccidental, lo que se traduce en gran cantidad de deformación intraplaca: con pliegues y cabalgamientos en la corteza superior y fallas transcurrentes con poco salto.

Las publicaciones científicas más recientes que tratan los campos de esfuerzos y deformaciones actuales del oeste de la placa Euroasiática a partir de los últimos datos de velocidades GPS indican que la corteza de la Península Ibérica al norte de la Cordillera Bética forma parte de Eurasia estable, al presentar una velocidad media residual de $0.3 \pm 0.1 \mathrm{~mm} / \mathrm{a}$ con respecto a la Europa estable (Nocquet et al. 2012), y no presenta deformaciones internas significativas dentro de la precisión de los datos GPS (Fernandes et al. 2007).

Por otro lado, Castilla - La Mancha es un área de amplio interés geológico, que incluye dominios geológicos diversos con evoluciones y características tectónicas distintas, como son las cuencas terciarias, Tajo y Guadalquivir, y los sistemas montañosos del Sistema Central, la Cordillera Ibérica, la parte meridional de la Cordillera Costero-Catalana, la Cordillera Bética Central y Oriental, el Prebético y partes significativas de los relieves antiguos del Macizo Hespérico como los Montes de Toledo, y Sierra Morena.

\subsubsection{Elaboración del catálogo de proyecto}

Para la elaboración del catálogo de proyecto se ha partido del confeccionado para el nuevo mapa de peligrosidad sísmica de España (MPSE) (IGN-UPM 2012), que contiene registros desde el año 1048 hasta el año 2011, y de una actualización de éste hasta el año 2016, en base al catálogo publicado por el Instituto Geográfico Nacional (IGN 2016).

\subsubsection{Homogeneización del catálogo}

Un catálogo sísmico es homogéneo, en lo referente al tamaño de sismos, cuando el parámetro que lo define es el mismo para todos los terremotos. Es imprescindible realizar las conversiones pertinentes entre las diferentes estimaciones de magnitud, a fin de obtener un catálogo sísmico homogéneo. En este trabajo se utiliza la magnitud momento $M_{w}$ como parámetro de tamaño, siguiendo la tendencia actual en estudios de amenaza sísmica.

Para homogeneizar las magnitudes a $\mathrm{M}_{\mathrm{w}}$, se tomaron en cuenta las correlaciones que se muestran en la Tabla 1.

Tabla 1: Correlaciones obtenidas entre distintos parámetros de tamaño de los sismos y la $\mathrm{M}_{\mathrm{w}}$ (regresiones RMA)

\begin{tabular}{c|c} 
Correlación & Rango de aplicación \\
\hline $\mathrm{M}_{\mathrm{w}}=1.656+0.545 \cdot \mathrm{I}_{\max }$ & III a IX $-\mathrm{X}$ \\
$\mathrm{M}_{\mathrm{w}}=0.290+0.973 \cdot \mathrm{m}_{\mathrm{bLg}(\mathrm{MMS})}$ & 3.1 a 7.3 \\
$\mathrm{M}_{\mathrm{w}}=-1.528+1.213 \cdot \mathrm{m}_{\mathrm{b}(\mathrm{VC})}$ & 3.7 a 6.3 \\
$\mathrm{M}_{\mathrm{w}}=0.676+0.836 \cdot \mathrm{m}_{\mathrm{bLg}(\mathrm{L})}$ & 3.0 a 5.1
\end{tabular}




\subsubsection{Depuración del catálogo}

La sismicidad sigue un modelo poissoniano, asumida en el método zonificado. Implica que la ocurrencia de cualquier terremoto es independiente de la ocurrencia de todos los demás, dentro de una misma fuente sísmica. En realidad, son frecuentes las asociaciones de terremotos en forma de series sísmicas, con un terremoto principal y otros premonitores y/o réplicas. Para verificar la hipótesis de independencia de sismos es necesario, entonces, detectar las series sísmicas e incluir en los cálculos únicamente los eventos principales, eliminando por tanto del catálogo los restantes de una misma serie.

Tras el proceso de depuración se identifican como réplicas un $36.9 \%$ de los registros, de modo que el catálogo depurado cuenta con un total de 7741 terremotos principales.

\subsubsection{Completitud}

Un catálogo sísmico se considera completo si contiene todos los sismos que han ocurrido en el área considerada. El problema es que al remontarnos hacia atrás en el tiempo, la información relativa a terremotos pequeños o no sentidos habitualmente se pierde.

El análisis de completitud consiste en identificar una serie de años de referencia que marcan el inicio del periodo de completitud, para cada zona y para cada rango de magnitudes. En la Tabla 2 se muestran los años de referencia considerados para cada rango de magnitud.

Tabla 2: Años de referencia considerados tras el estudio de completitud

\begin{tabular}{c|c|c}
$\begin{array}{c}\text { Rango de } \\
\text { magnitudes }\end{array}$ & $\begin{array}{c}\text { Catálogo } \\
\text { MPSE }\end{array}$ & $\begin{array}{c}\text { Catálogo SE de } \\
\text { España }\end{array}$ \\
\hline $3.0-3.4$ & 1985 & 1978 \\
$3.5-3.9$ & 1980 & 1975 \\
$4.0-4.4$ & 1933 & 1908 \\
$4.5-4.9$ & 1910 & 1883 \\
$5.0-5.4$ & 1800 & 1800 \\
$5.5-5.9$ & 1720 & 1520 \\
$6.0-6.4$ & $\ldots$ & $\ldots$ \\
$\geq 6.5$ & $\ldots$ & $\ldots$
\end{tabular}

\subsubsection{Catálogo final de proyecto}

En la Tabla 3 se muestran las principales características del catálogo empleado en el presente estudio (Fig. 2).

Tabla 3: Características principales del catálogo empleado en el estudio

\begin{tabular}{c|c|c|c|c}
$\begin{array}{c}N^{\circ} \text { de } \\
\text { eventos }\end{array}$ & $\begin{array}{c}\text { Periodo } \\
\text { temporal }\end{array}$ & $\begin{array}{c}\text { Tipo de } \\
\text { magnitud }\end{array}$ & $\begin{array}{c}\text { Rango de } \\
\text { magnitudes }\end{array}$ & $\begin{array}{c}\text { Rango de } \\
\text { profundidad } \\
(\mathrm{km})\end{array}$ \\
\hline 7741 & $\begin{array}{c}1048- \\
2016\end{array}$ & $\begin{array}{c}\text { Momento } \\
\left(\mathrm{M}_{\mathrm{w}}\right)\end{array}$ & $3.0-8.5$ & $0-65$ \\
& & &
\end{tabular}

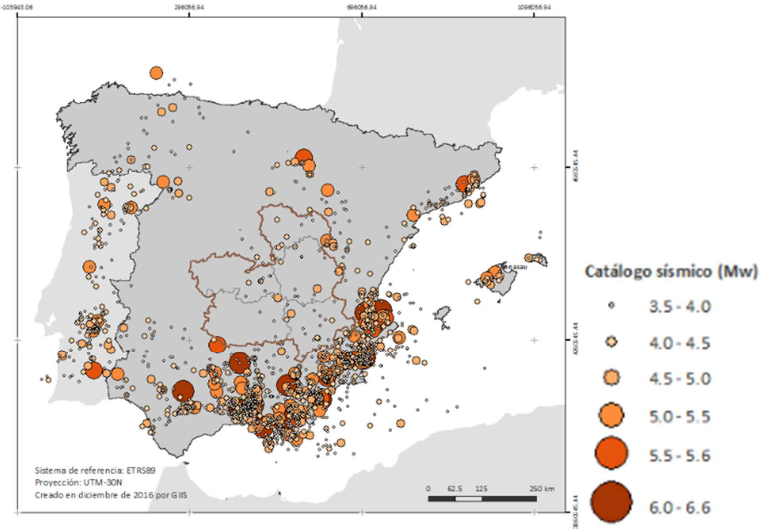

Figura 2: Mapa con la distribución de los sismos considerados en el catálogo de estudio.

\subsubsection{Identificación y caracterización de fuentes sísmicas en el área de estudio}

Para el cálculo de peligrosidad sísmica se tienen en cuenta dos fuentes generadoras de sismos: fallas activas y zonas sismogenéticas.

Para incluir ambos tipos de fuente en el cálculo de peligrosidad, debe caracterizarse su patrón de sismicidad o ley de recurrencia, junto con la magnitud máxima (Mmax) que cada fuente pueda generar.

\subsubsection{Fallas activas en el área de estudio}

El movimiento brusco en una falla activa genera una liberación de energía que se propaga en forma de ondas. Cuando éstas alcanzan la superficie terrestre, se produce la sacudida sísmica conocida como terremoto.

Para este trabajo se han tenido en cuenta tanto las fallas incluidas en la base de datos de fallas activas del Cuaternario para la Península Ibérica, QAFI v.3 (GarcíaMayordomo et al. 2012a) como las fallas incluidas en el mapa neotectónico de la Península lbérica (IGME, ENRESA 1998).

En la Comunidad Autónoma de Castilla-La Mancha son muy escasas las fallas con evidencias geológicas directas de actividad cosísmica cuaternaria. No obstante, para el estudio del riesgo sísmico se han tenido en cuenta también las fallas activas que, aun quedando fuera de los límites de esta Comunidad Autónoma, están dentro de la zona de influencia considerada en el estudio (radio de $300 \mathrm{~km}$ en torno al centroide de Castilla - La Mancha) (Fig. 3).

\section{Caracterización sísmica de las fallas}

La actividad sísmica de las fallas viene dada por el sismo máximo que se espera en cada una y por la tasa de momento sísmico que se acumule, suponiendo que la energía se acumula de forma homogénea en todo el plano de falla.

La magnitud máxima esperada en la falla (o segmento) se obtiene a partir del área del plano de falla o superficie de ruptura $(A)$, considerando que la Mmax es proporcional a $A$. Se emplean las correlaciones propuestas en la literatura: Wells and Coppersmith (1994), Stirling et al. (2002) y Stirling et al. (2008). Se consideran valores promedio entre los estimados con las diferentes correlaciones. 
En relación a la tasa de momento acumulada, se supone que en todo el plano de falla se está acumulando energía uniformemente y la tasa de momento sísmico Mo que se acumula en la falla (moment rate) está relacionada con la tasa de deslizamiento anual de la misma $\dot{u}$ (slip rate) según la ecuación de Brune (1968), Ec. (4):

$\dot{M}_{0}=\mu \cdot \dot{u} \cdot A$

Donde:

$\mu$ es el módulo de rigidez o de cizalla.

$\dot{u}$ es la tasa de deslizamiento.

$A$ es el área del plano de falla.

\subsubsection{Zonificación sísmica}

Además de considerar las fallas como fuentes generadoras de sismos se tienen en cuenta zonas sismogenéticas para así contemplar la sismicidad que no está claramente asociada a las fallas identificadas. Estas zonas se consideran con potencial sísmico homogéneo y a cada una de ellas se asocia un patrón de sismicidad definido.

Se ha considerado la zonificación de GarcíaMayordomo (2010), desarrollada a partir de la reunión científica IBERFAULT 2010. Esta zonificación aporta una visión geológica general de la región de estudio y considera criterios sísmicos, geológicos, tectónicos y reológicos a la hora de la definición y caracterización de las zonas, dando distintos pesos a cada tipo de dato. La zonificación aporta además, parámetros de actividad de las fallas activas a partir de datos geológicos, cuando éstos datos están disponibles.

Se han considerado aquellas zonas que intersecan con el área de influencia del estudio (Fig. 3).

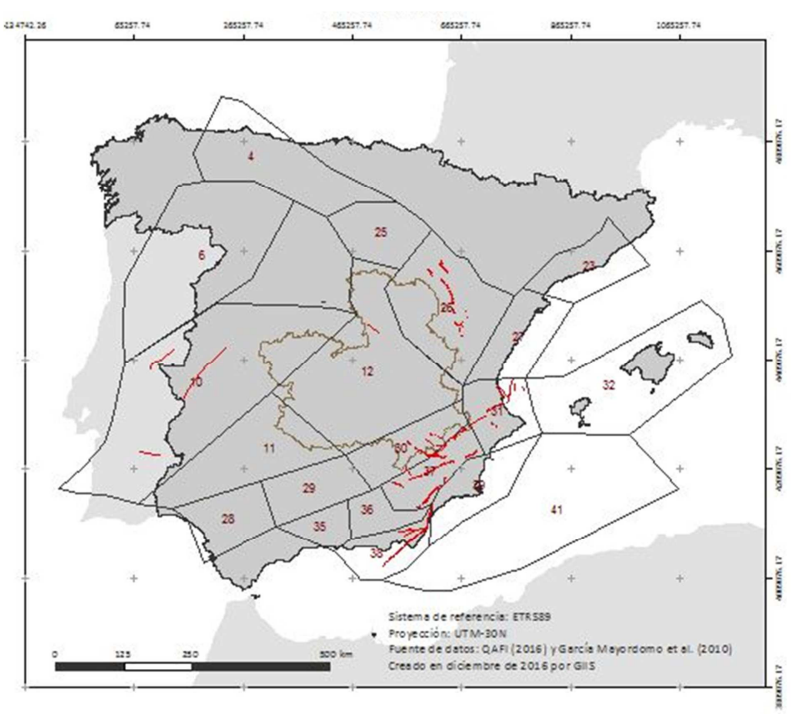

Figura 3: Zonificación de lberfault (2010) y fallas consideradas en el estudio de Castilla - La Mancha (fuente: elaboración propia).

\section{Caracterización sísmica de las zonas}

Dentro de cada zona sismogenética, la sismicidad se distribuye de manera aleatoria espacial y temporalmente. Se requiere entonces deducir la ley de recurrencia de sismos en función de su magnitud, así como la magnitud máxima creíble en la zona.

Para ello, se analiza la distribución de sismos en la zona de estudio, asumiendo el modelo de Gutenberg and Richter (1944) que es el modelo habitualmente empleado en cálculos de peligrosidad siguiendo métodos zonificados, Ec. (5). Este modelo establece que el logaritmo del número de terremotos, $N$, cuya magnitud es igual o mayor a una magnitud dada, $m$, está relacionado linealmente con esa magnitud:

$\log N=a+b \cdot m$

Donde:

$b$ es una estimación de la proporción entre terremoyos grandes y pequeños que ocurren en la zona.

$a$ es un valor que está relacionado con el número de terremotos que supera una magnitud mínima Mo.

Por otro lado, del catálogo sísmico se puede deducir la Mmax observada en la zona, pero esta puede ser inferior a la del sismo máximo que pueda ser generado en las fallas existentes. De hecho, el catálogo sísmico suele no cubrir ciclos completos de dichas fallas, sobre todo cuando éstas son fallas lentas como las localizadas en el sureste de España. Por tanto la Mmax correspondiente al máximo sismo que pueda generarse puede ser superior a la Mmax observada en el catálogo. Para tener en cuenta esta incertidumbre sobre la Mmax de cada zona, se ha considerado una distribución Gaussiana (Fig. 4) definida por los parámetros:

- $\quad$ Mmax ( $\min )$ magnitud máxima observada en la zona derivada del catálogo sísmico que se identifica con el umbral mínimo de la distribución.

- Mmax (max) magnitud máxima acorde con la geología de la zona (Mmax (max)), identificada con el límite máximo de la distribución.

- $\quad \mathrm{E}(\mathrm{Mmax}$ (media)) magnitud máxima esperada.

- $\sigma$ desviación estándar de la distribución de la Mmax.

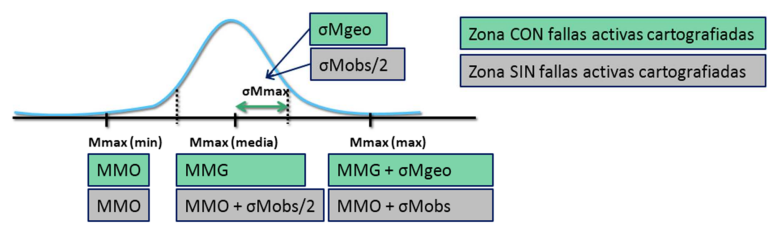

Figura 4: Esquema de definición de las magnitudes de las zonas sismogenéticas (fuente: elaboración propia).

\subsubsection{Leyes de atenuación o modelos de movimiento fuerte}

Tras la caracterización de las fuentes sísmicas, el segundo factor determinante en el cálculo de peligrosidad es la atenuación que experimentan las ondas sísmicas en su recorrido desde la fuente a cada emplazamiento de estudio. Para considerar este efecto deben aplicarse leyes de atenuación, también conocidas como ecuaciones de predicción del movimiento o 
GMPEs (del inglés Ground Motion Prediction Equations).

Estas ecuaciones permiten estimar el desplazamiento causado en un cierto emplazamiento por el movimiento generado en la fuente, a una cierta distancia del mismo, proporcionado valores del parámetro característico del movimiento para una magnitud y distancia dadas.

Para seleccionar los modelos más idóneos para su aplicación en Castilla - La Mancha, entre los propuestos en la literatura especializada, se efectúa un contraste de modelos con datos locales, que permita determinar cuáles de esos modelos reproducen mejor la atenuación de nuestra zona. En la Tabla 4 se muestran las características principales de los modelos empleados.

Tabla 4: Características principales de los modelos de atenuación empleados en el estudio

\begin{tabular}{c|c|c} 
& $\begin{array}{c}\text { Abrahamson et al. } \\
\text { (2013) (ASK 2013) }\end{array}$ & $\begin{array}{c}\text { Campbell and } \\
\text { Bozorgnia (2013) } \\
\text { (CB13) }\end{array}$ \\
\hline $\begin{array}{c}\text { Base de datos } \\
\text { Componente } \\
\text { utilizada para } \\
\text { (PGA, SA(T)) }\end{array}$ & Mundial & Mundial \\
Tipo de fuente & Cortical & Media geométrica \\
Distancia $(\mathrm{km})$ & $0-300$ & $0-300$ \\
Mw & $3.0-8.5$ & $3.0-8.5$
\end{tabular}

\subsubsection{Efecto local}

La naturaleza y la distribución de los daños producidos por la ocurrencia de un terremoto están influenciados por la respuesta del terreno frente a cargas cíclicas. Como consecuencia, se produce una modificación de la señal sísmica, denominada "efecto de sitio". Dicha respuesta y la magnitud del daño producido están controladas principalmente por las condiciones geológico-geotécnicas de las formaciones geológicas, especialmente por las características dinámicas del terreno.

Por regla general, la presencia de terrenos blandos no consolidados de gran potencia produce un aumento considerable de los daños que se generan en las infraestructuras situadas sobre dichos suelos ante la ocurrencia de un sismo.

La región de Castilla - La Mancha presenta una gran variedad de litologías de distinta génesis y edad. Esta variabilidad litológica da lugar a materiales de diferente resistencia mecánica, que van desde rocas muy duras y competentes, hasta terrenos blandos y muy blandos.

Se han delimitado 9 grupos de materiales en base a las propiedades geológico-geotécnicas y posteriormente, a cada uno de estos grupos, se le ha asignado el grado de amplificación de las ondas sísmicas a partir del valor de velocidad de ondas de cizalla (Vs). Para ello, se ha empleado la normativa FEMA (FEMA 2003). Se ha obtenido así un mapa de clases de con diferente grado de amplificación sobre el movimiento en roca (Fig. 5). En la Tabla 5 se muestra un resumen de las características principales de cada una de estas clases.

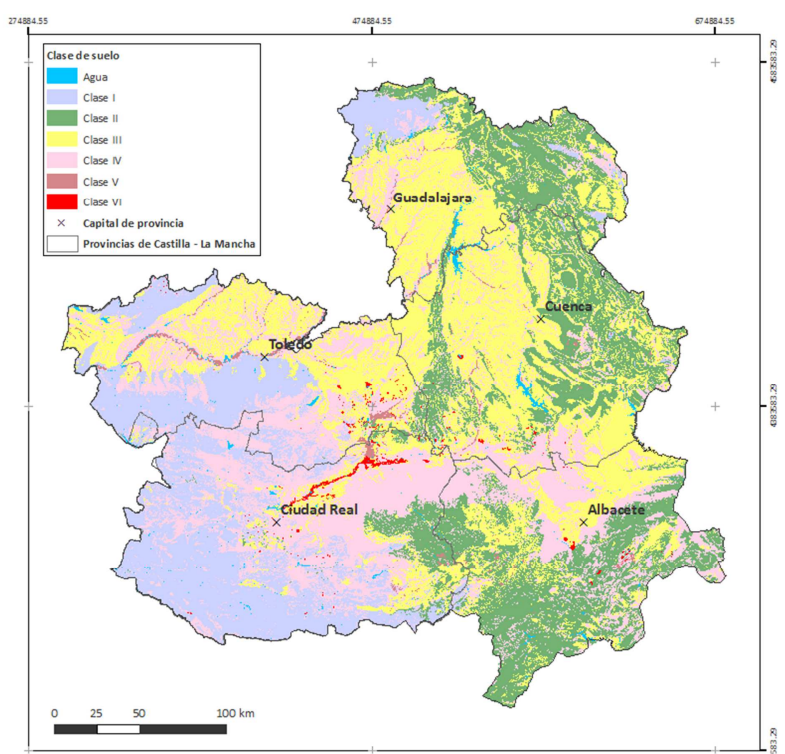

Figura 5: Mapa de clases de suelos localizados en Castilla La Mancha (fuente: elaboración propia) (fuente: elaboración propia).

Tabla 5: Resumen de las características principales de las clases de suelos localizadas en Castilla - La Mancha

\begin{tabular}{c|c|c|c} 
Clase & Descripción geotécnica & $\begin{array}{c}\text { Vs } \\
(\mathrm{m} / \mathrm{s})\end{array}$ & $\begin{array}{c}\text { Tipo suelo } \\
\text { NEHRP }\end{array}$ \\
\hline I & $\begin{array}{c}\text { Roca muy dura, poco } \\
\text { fracturada }\end{array}$ & $>1500$ & $\mathrm{~A}$ \\
II & $\begin{array}{c}\text { Roca dura media, } \\
\text { fracturada. }\end{array}$ & $751-$ & $\mathrm{B}$ \\
III & $\begin{array}{c}\text { Roca de resistencia } \\
\text { blanda, muy fracturada. }\end{array}$ & 351 & $\mathrm{C}$ \\
IV & $\begin{array}{c}\text { Suelos no cohesivos, poco } \\
\text { compactos. }\end{array}$ & $180-$ & $\mathrm{C}$ \\
V & 350 & $\mathrm{D}$ \\
VI & $\begin{array}{c}\text { Suelos cohesivos blandos. } \\
\text { Suelos muy blandos, } \\
\text { orgánicos. }\end{array}$ & $<100$ & $\mathrm{E}$ \\
& $<100$ & $\mathrm{~F}$
\end{tabular}

\subsubsection{Resultados de peligrosidad sísmica con efecto de sitio}

En las Figuras 6 y 7 se muestran los resultados de peligrosidad sísmica, considerando el efecto local, en términos de PGA para PR de 475 años y 975 años, respectivamente.

\section{Exposición de las edificaciones y población}

El Instituto Nacional de Estadística (INE) proporciona información sobre el número de edificios por municipio, así como las características necesarias para un estudio de riesgo sísmico (año de construcción, número de plantas, uso, superficie construida...). Los datos sobre la vivienda publicados por la institución están actualizados hasta el año 2011. En total, en Castilla - La Mancha, y hasta la fecha mencionada, hay registrados 842607 edificios. 


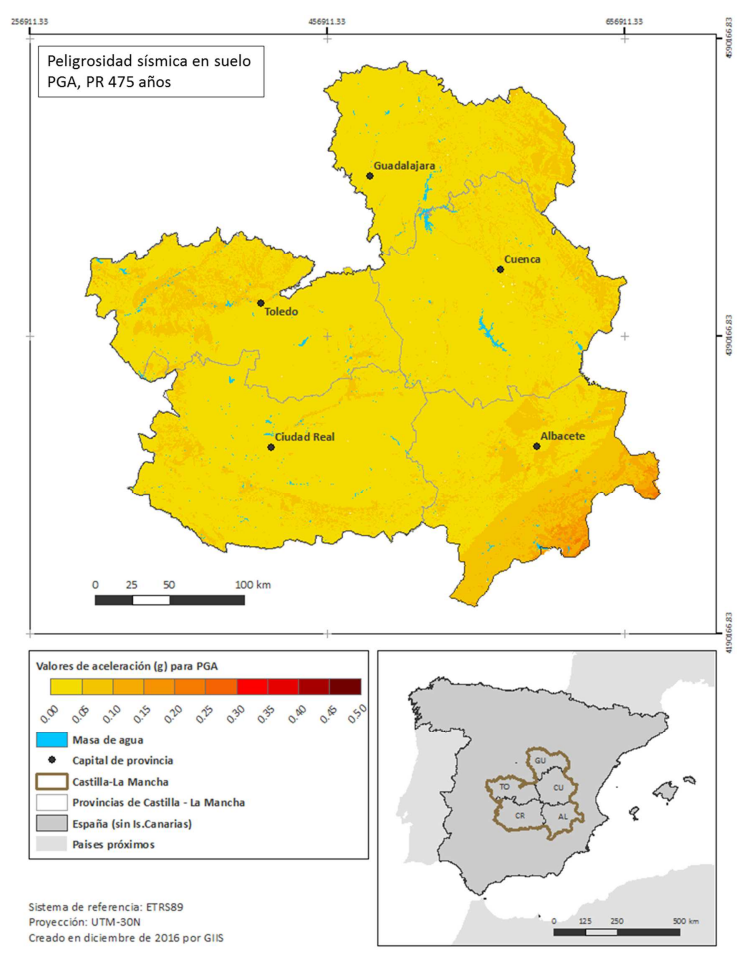

Figura 6: Mapa de peligrosidad sísmica con efecto local de la Comunidad de Castilla - La Mancha (PGA, PR 475 años) (fuente: elaboración propia).

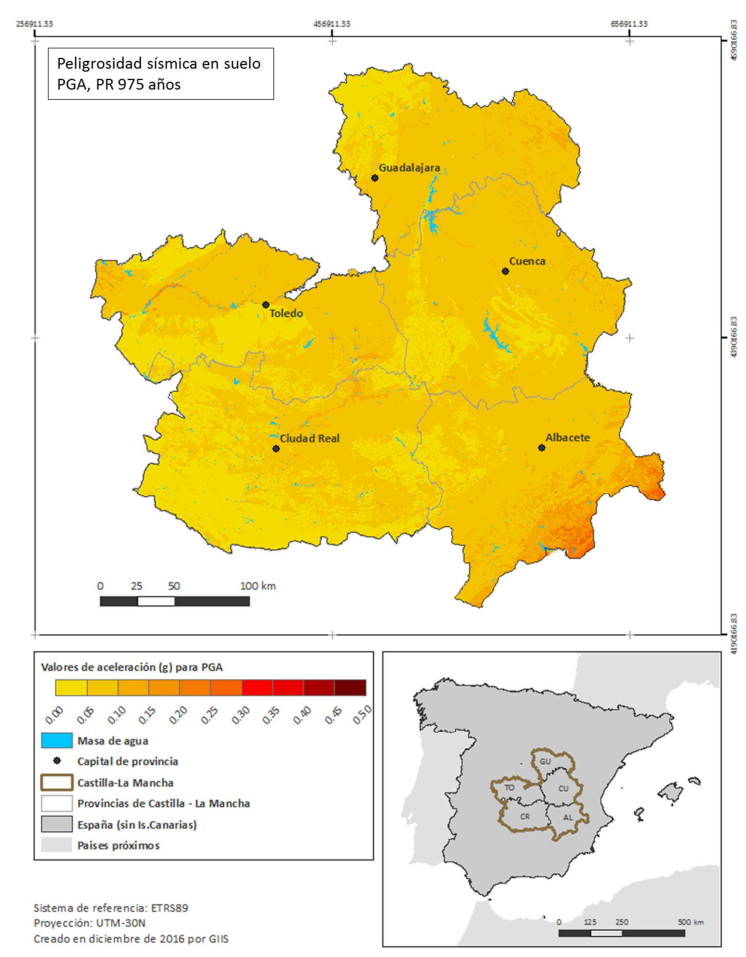

Figura 7: Mapa de peligrosidad sísmica con efecto local de la Comunidad de Castilla - La Mancha (PGA, PR 975 años) (fuente: elaboración propia).

De igual manera, a partir de la información que facilita el INE, es posible conocer el número habitantes por municipio. A fecha de 1 de enero de 2015, en Castilla La Mancha, hay un total de 2062767 habitantes y la densidad media es de 26 habitantes por kilómetro cuadrado.

Castilla - La Mancha posee un parque inmobiliario muy heterogéneo, compuesto por edificios antiguos y otros modernos. Se identifican dos grandes grupos de edificaciones de uso residencial:

- Edificaciones tradicionales. Son edificios que se apoyan en conceptos de buena práctica constructiva realizada con anterioridad a las décadas de los 40 o 50.

- Edificación tecnológica. Son construcciones realizadas con un procedimiento de cálculo donde los esfuerzos sobre la estructura son previstos y se desarrollan soluciones específicas para ello. La implantación de la etapa tecnológica sucede a lo largo de varias décadas, pero es útil relacionarla con la publicación de las primeras normas sismorresistentes españolas, que entraron en vigor a partir del año 1962.

\subsection{Asignación de vulnerabilidad}

El término vulnerabilidad se define como la fragilidad de una edificación ante una acción sísmica. Esta fragilidad viene determinada por las prestaciones sismorresistentes accidentales o proyectadas que posee la estructura, y éstas a su vez, por las características tecnológicas de su composición.

La clasificación de vulnerabilidad de un parque inmobiliario obliga a determinar las tipologías constructivas de los edificios que lo componen, determinar sus prestaciones sismorresistentes, y clasificarlas según la respuesta que puedan tener ante el sismo, todo ello avalado por la literatura científica.

En la Tabla 6 se muestran los principales tipos constructivos localizados en Castilla - La Mancha.

Tabla 6: Tipos constructivos para viviendas más frecuentes localizados en Castilla - La Mancha

\begin{tabular}{c|c} 
Código & Descripción \\
\hline MPFM & Estructura muraria de piedra sin forjados rígidos \\
MAFM & $\begin{array}{c}\text { Estructura muraria de adobe sin forjados rígidos } \\
\text { MLFM }\end{array}$ \\
ELFH & $\begin{array}{r}\text { Estructura muraria de fábrica de ladrillo sin } \\
\text { forjaria des rígidos } \\
\text { forjados rígidos con efecto diafragma de ladrillo con }\end{array}$ \\
HPAL & $\begin{array}{r}\text { Estructura de pórticos de hormigón armado con } \\
\text { cerramientos de albañilería }\end{array}$
\end{tabular}

Para asignar clases de vulnerabilidad a las tipologías identificadas en el parque inmobiliario de Castilla - La Mancha se considera la clasificación propuesta en la metodología del proyecto RISK-UE (Milutinovic and Trendafiloski 2003). En la Tabla 7 se muestra la correlación entre las tipologías más representativas para viviendas localizadas en la región de estudio y las consideradas en dicha metodología. Esta correlación se lleva a cabo atendiendo al tipo constructivo y al número de plantas de las edificaciones. 
Tabla 7: Correlación entre las tipologías localizadas en Castilla - La Mancha para viviendas y la clasificación de vulnerabilidad según RISK-UE (Milutinovic and Trendafiloski 2003)

\begin{tabular}{c|c|c|c} 
Código & 1 - 2 plantas & $3-5$ plantas & $\geq 6$ plantas \\
\hline MPFM & M1.1 LOW & M1.1 MED & $\ldots$ \\
MAFM & M1.1 LOW & M1.1 MED & $\ldots$ \\
MLFM & M3.4 LOW & M3.4 MED & M3.4 HIGH \\
MLFH & M3.4 LOW & M3.4 MED & M3.4 HIGH \\
HPAL & RC3.1 LOW & RC3.1 MED & RC3.1 HI
\end{tabular}

Además, es necesario conocer el nivel de diseño sismorresistente de las estructuras. Se considera que las estructuras murarias no tienen asociado ningún nivel de diseño. Sin embargo, para las estructuras de hormigón armado, definidas como RC3.1 según RISK UE, es necesario analizar el desarrollo histórico de las normas sismorresistentes de aplicación en Castilla-La Mancha desde 1962 hasta la actualidad.

Finalmente, se considera que las estructuras (RC3.1) construidas entre 1941 y 1994 no tienen asociado ningún nivel de diseño sismorresistente (pre-code) y aquellas que se construyeron a partir de 1995 tienen asociado un nivel de diseño bajo (low-code).

A modo de resumen, en la Figura 8 se muestra la distribución de tipologías estimada para toda Castilla La Mancha.

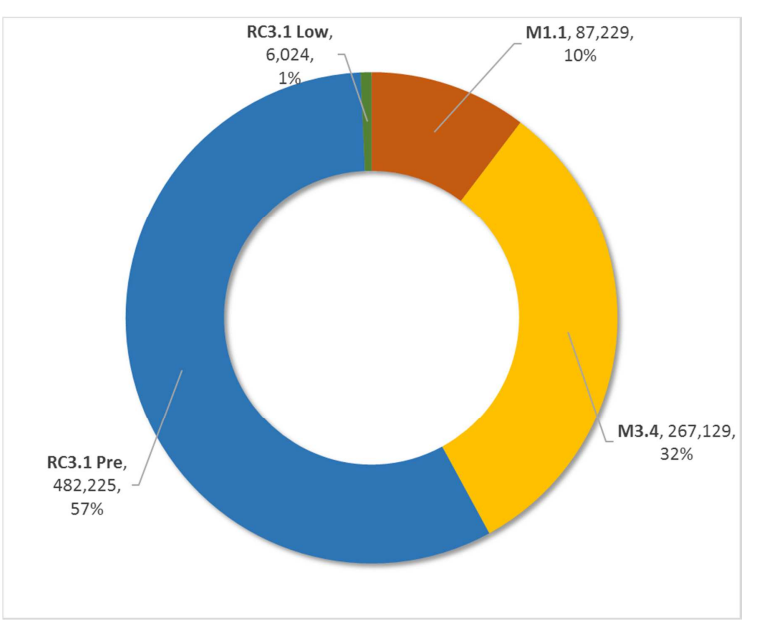

Figura 8: Distribución de la vulnerabilidad del parque inmobiliario (viviendas) de Castilla - La Mancha (fuente: elaboración propia).

\section{Base de datos georreferenciada}

Como se ha mencionado anteriormente, es necesario crear un SIG que permita gestionar toda la información geográfica que compone la base de datos del proyecto. Esta base de datos debe incluir, al menos, la siguiente información geográfica:

- Zonas sismogenéticas.

- Fallas activas.

- Catálogo sísmico.

- Datos administrativos: distritos, secciones censales, límites municipales.
- Elementos expuestos:

- Datos catastrales: valor catastral, número de edificios y viviendas, superficie construida, etc.

- Datos de población y ocupación de viviendas

Como unidad de trabajo y representación para los resultados de riesgo sísmico se considera cada uno de los municipios que componen la Comunidad Autónoma (919 municipios).

Para la realización de este estudio se fija como sistema de referencia el ETRS89 (Sistema de Referencia Terrestre Europeo 1989), coincidiendo con el sistema de referencia oficial de España, tal y como aparece publicado en el Boletín Oficial del Estado (BOE) número 207 (BOE 2007). Para la presentación final de resultados, se emplea la proyección UTM (Universal Transversa de Mercator) en el Hemisferio Norte Huso 30.

\section{Evaluación del riesgo sísmico}

\subsection{Cálculo del daño esperado: Método I- DCM}

En este estudio se emplea un procedimiento analítico estático no lineal para estimar el daño en los edificios, de acuerdo al movimiento del suelo esperado dado en términos de aceleraciones y desplazamientos espectrales.

Partiendo de la clasificación de los edificios por tipología estructural, con su correspondiente clase de vulnerabilidad asignada, se calcula la probabilidad de que una clase alcance o supere diferentes grados de daño a partir del movimiento máximo esperado en la unidad de estudio.

Para caracterizar la respuesta de las estructuras, se aplica el método de capacidad-demanda. En este método la demanda se identifica con la acción sísmica (mediante el espectro de respuesta) y la capacidad viene dada por la curva que refleja el comportamiento de la estructura (desde el régimen lineal hasta el punto de ruptura). Se determina entonces el punto de desempeño, que corresponde al desplazamiento espectral que se espera que sufra cada tipo de estructura a causa del movimiento sísmico de entrada (demanda). Para calcular el punto de desempeño se emplea el método del coeficiente de desplazamiento mejorado, I-DCM (FEMA 2005), que consiste en modificar la demanda de desplazamiento del sistema lineal de un grado de libertad (SDOF) equivalente a la estructura multiplicándolo por una serie de coeficientes para estimar el desplazamiento máximo del oscilador no lineal.

Por último, se emplean curvas de fragilidad que proporcionan la probabilidad de alcanzar o exceder diferentes grados de daño en función del desplazamiento espectral para el punto de desempeño (Fig. 9). 


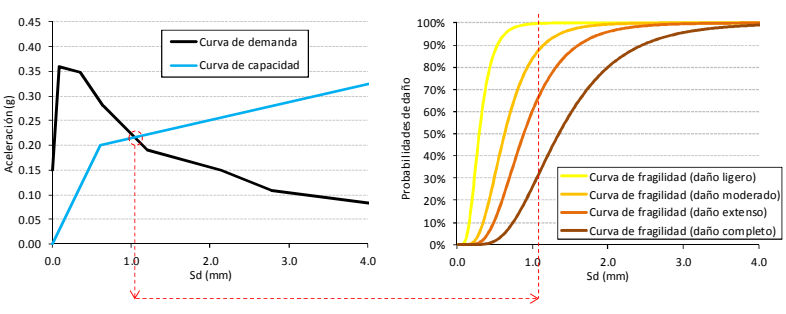

Figura 9. Esquema del método de cálculo de capacidad demanda (fuente: elaboración propia).

\subsubsection{Clasificación y estimación de grados de daño}

La clasificación de los grados de daño se basa en la metodología propuesta en RISK-UE (Milutinovic and Trendafiloski 2003). Esta metodología considera una clasificación atendiendo a daños estructurales y no estructurales en edificios de importancia normal, viviendas convencionales (Tabla 8 ).

Tabla 8: Grados de daño según RISK-UE Milutinovic y Trendafiloski 2003)

\begin{tabular}{c|c|c|c}
$\begin{array}{c}\text { Grado } \\
\text { de daño }\end{array}$ & $\begin{array}{c}\text { Descripción } \\
\text { cualitativa }\end{array}$ & $\begin{array}{c}\text { Daño } \\
\text { estructural }\end{array}$ & $\begin{array}{c}\text { Daño no } \\
\text { estructural }\end{array}$ \\
\hline 0 & Nulo & $\begin{array}{c}\text { Sin Daños } \\
\text { Insignificante a } \\
\text { Ligero }\end{array}$ & $\begin{array}{c}\text { Sin daños } \\
\text { Ligero }\end{array}$ \\
1 & Leve & Ligero & Moderado \\
2 & Moderado & Moderado & Severo \\
3 & Extenso & Suy Severo a & $\begin{array}{c}\text { Muy Severo a } \\
\text { Colapso }\end{array}$ \\
4 & Severo & $\begin{array}{c}\text { Colapso } \\
\end{array}$
\end{tabular}

\subsection{2. Índice de daño medio}

El índice de daño medio $(\mathrm{Dm})$ es un parámetro de medida del daño en edificios. A través de este índice, se establece el promedio de los daños ocasionados en las estructuras ante la ocurrencia de un evento sísmico. Es un parámetro que varía entre cero y cuatro, siendo cero el equivalente a daño nulo y cuatro el equivalente a daño completo o colapso (Hazus-MH 2003). A partir de la Ec. (6) es posible calcular el Dm.

$D m=0 \times D_{n}+1 \times D_{l}+2 \times D_{m}+3 \times D_{e}+4 \times D_{c}$

Donde:

$$
\begin{aligned}
& D_{n} \text { es el daño nulo. } \\
& D_{l} \text { es el daño ligero. } \\
& D_{m} \text { es el daño moderado. } \\
& D_{e} \text { es el daño extenso. } \\
& D_{c} \text { es el daño completo. }
\end{aligned}
$$

\subsubsection{Otros parámetros de riesgo}

A partir de los daños físicos registrados en las viviendas es posible determinar otros parámetros de riesgo como las pérdidas humanas y pérdidas económicas.

La estimación de pérdidas humanas se realiza siguiendo el método empírico de Coburn and Spence (2002), en función del número de edificios cuyo estado de daño resulte completo (sólo los que se estiman que colapsarían), la densidad de población y la intensidad del movimiento esperado.

Por otro lado, para llevar a cabo la estimación de los costes de reconstrucción o de reparación de las estructuras dañadas (sin tener en cuenta el valor del material contenido en las viviendas) es necesario conocer el valor de construcción por metro cuadrado y la superficie promedio de la vivienda en la zona de estudio.

\subsubsection{Resultados de riesgo sísmico}

Los resultados de riesgo se han obtenido para cada uno de los municipios de Castilla - La Mancha, representados en mapas que contienen información de distintos parámetros indicativos de las pérdidas esperadas. A continuación, se muestran a modo de ejemplo algunos de los mapas resultantes del presente estudio.

Las Figuras 10, 11, 12, 13 y 14 muestran el índice de daño medio para cada municipio de cada una de las provincias de la Castilla - La Mancha. Los resultados corresponden a un PR de 475 años.

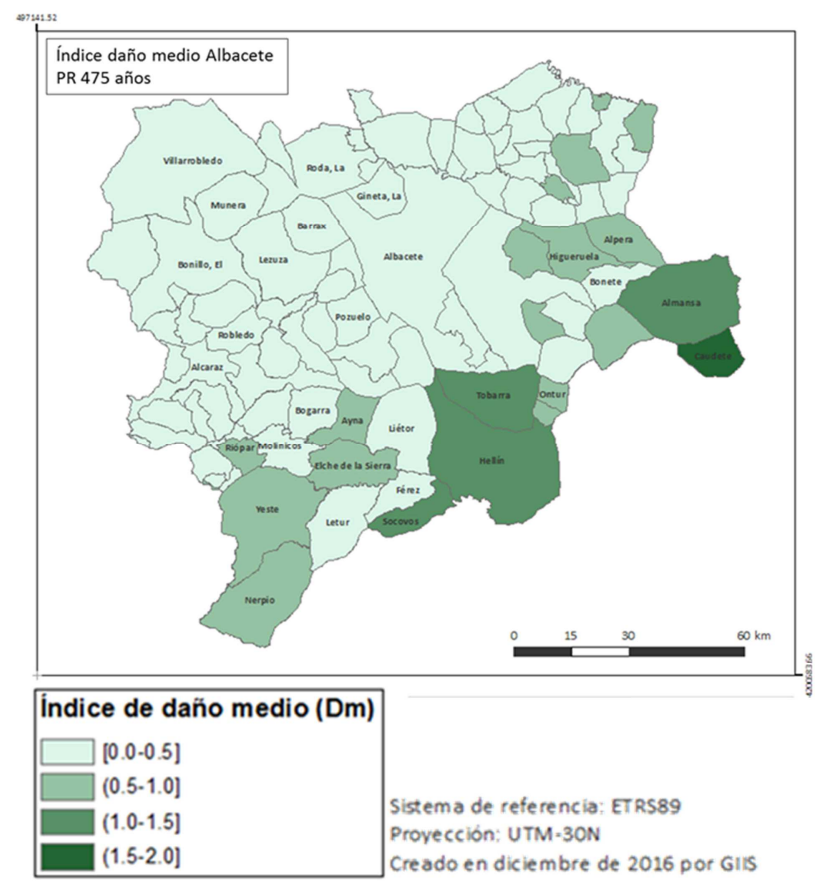

Figura 10. Mapa del índice de daño medio en edificios de viviendas para los municipios de la provincia de Albacete (PR 475 años).

\section{Discusión de resultados, conclusiones y recomendaciones}

En base al estudio de las características geotécnicas de los materiales geológicos, se concluye que en Castilla La Mancha dichos materiales presentan poca posibilidad de amplificación. Los materiales que pueden amplificar las ondas sísmicas en superficie (clases III-VI) ocupan el $60 \%$ del total de materiales que afloran en Castilla - La Mancha. Dentro de éstos, el 2,1\% pertenece a materiales de amplificación alta o muy alta. Estos últimos se encuentran próximos a pequeños núcleos de población, como los municipios de Alcázar de San Juan, 
Villarrubia de los Ojos, Pedro Muñoz, Lillo y Tembleque, todos ellos pertenecientes a las provincias de Toledo y Ciudad Real.

Los resultados de peligrosidad sísmica con efecto local (Figs. 6 y 7) muestran que para un PR de 475 años los valores máximos de aceleración serían de $0.22 \mathrm{~g}$ y para el periodo de retorno de 975 años de $0.28 \mathrm{~g}$.

Del número total de edificios de Castilla-La Mancha, el $42 \%$ pertenece a tipologías tradicionales de edificación, (M1.1 y M3.4) mientras que el restante $58 \%$ del parque inmobiliario corresponden a edificación tecnológica. Una pequeña proporción del parque inmobiliario, (el 1\%) desarrolla prestaciones sismorresistentes por aplicación de las normas NCSE 94 y NCSE 02, y las edificaciones correspondientes se asignan a la tipología RC3.1 Low Code.

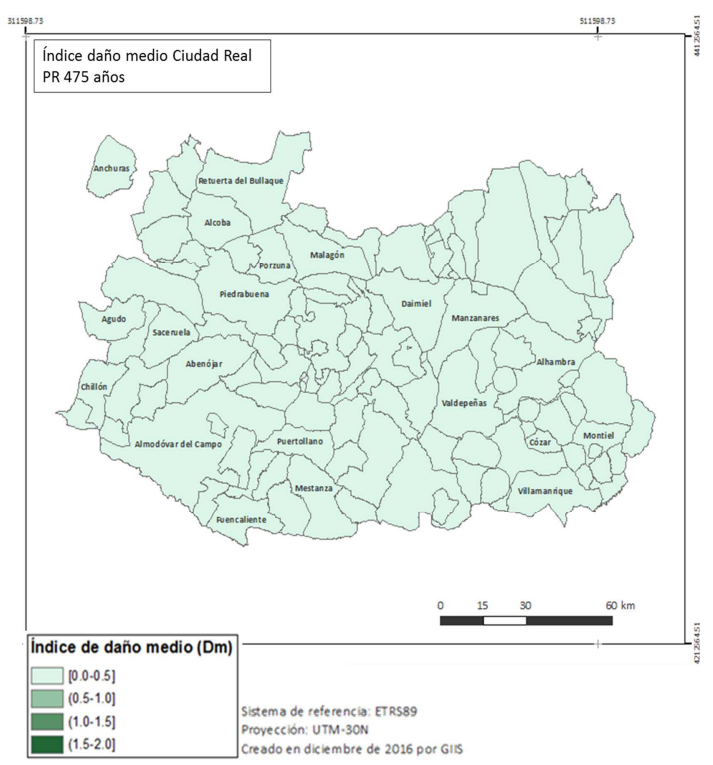

Figura 11. Mapa del índice de daño medio en edificios de viviendas para los municipios de la provincia de Ciudad Real (PR 475 años).

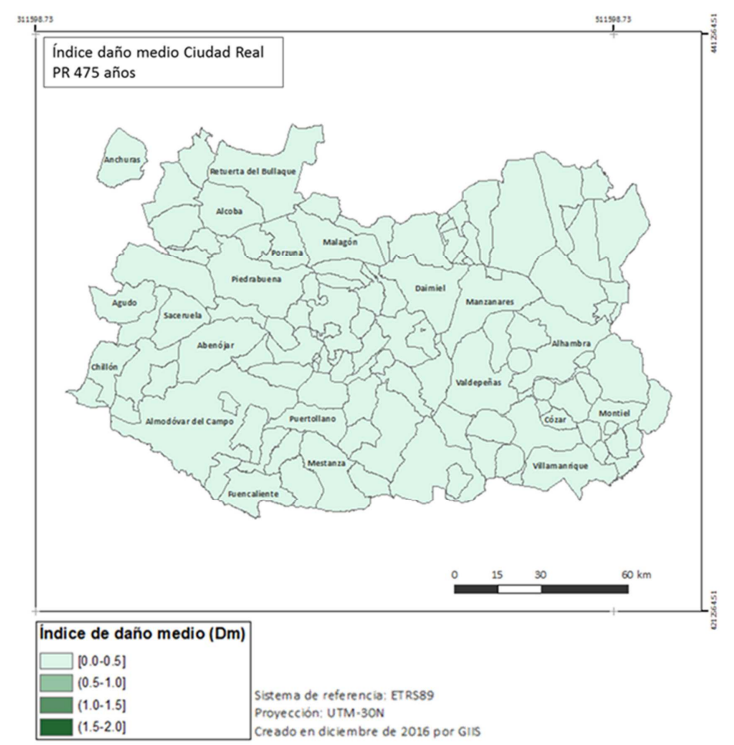

Figura 12. Mapa del índice de daño medio en edificios de viviendas para los municipios de la provincia de Cuenca (PR 475 años).

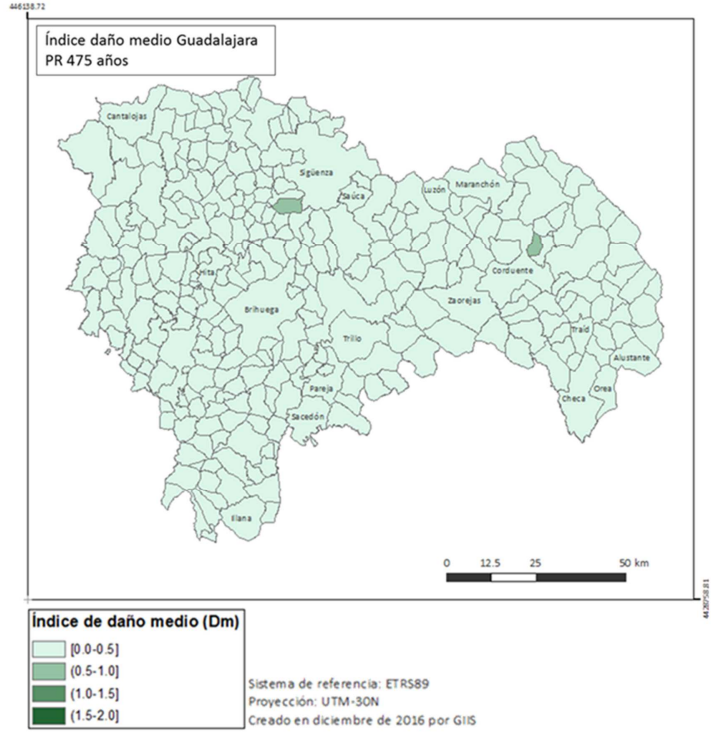

Figura 13. Mapa del índice de daño medio en edificios de viviendas para los municipios de la provincia de Guadalajara (PR 475 años).

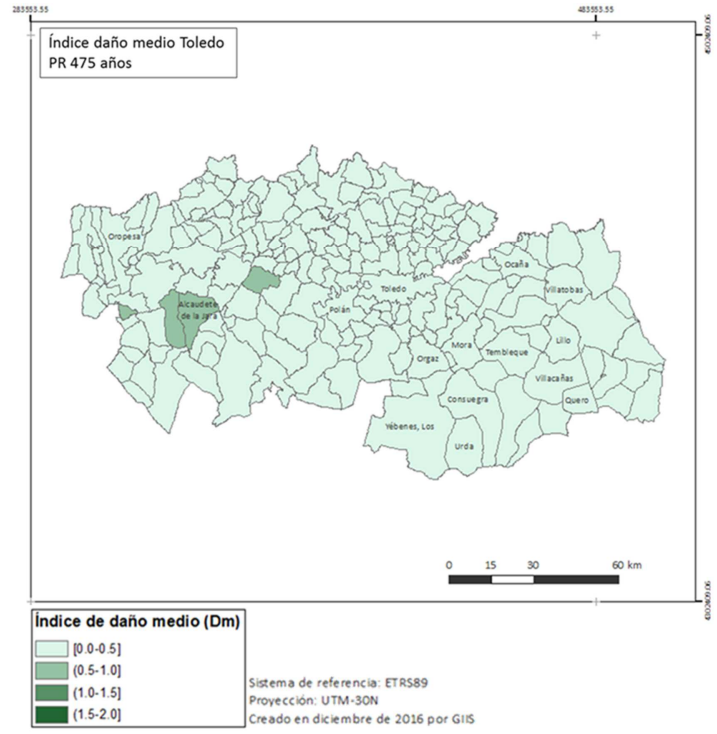

Figura 14. Mapa del índice de daño medio en edificios de viviendas para los municipios de la provincia de Toledo (PR 475 años).

Con respecto al índice de daño medio (Figs. 10, 11, 12, 13 y 14) se puede observar que, como máximo, se alcanzan valores cercanos a 2 en la provincia de Albacete, correspondiente a un Daño Moderado. La mayoría de los municipios de la Comunidad muestran valores inferiores a 1, lo que indica que la mayor parte de los edificios de la Castilla - La Mancha no se verán afectados, o tendrán únicamente daños ligeros, por los movimientos sísmicos esperados con probabilidad de excedencia del $10 \%$ en 50 años.

A partir de los resultados obtenidos se considera adecuado el cálculo de la peligrosidad sísmica con un enfoque probabilista - determinista, definiendo escenarios sísmicos mas concretos y efectuando el posterior cálculo del riesgo sísmico, para la provincia de Albacete (provincia con resultados mayores de daño). 
Los resultados del estudio se han facilitado a Protección Civil de Castilla La Mancha en dos tipos de herramientas:

- Un SIG, que permite el almacenamiento y gestión de los datos y resultados derivados de cada fase del trabajo.

- Un visualizador que facilita el uso de la información geográfica a profesionales de diversas áreas que no necesariamente tienen amplios conocimientos de SIG.

Las dos herramientas permiten que las instituciones destinadas a la elaboración de planes de emergencias establezcan prioridades de actuación, dimensiones los recursos para abordar la emergencia en cada municipio y tomen las decisiones oportunas para mitigación del riesgo.

\section{Agradecimientos}

Por un lado, agradecimiento especial al grupo de investigación "Geodinámica planetaria, tectónica activa y aplicaciones a riesgos" de la Universidad Complutense de Madrid por su gran colaboración en referencia al análisis de la tectónica y sismicidad de la zona, la estimación de las características geotécnicas de la zona de estudio y la definición del efecto local.

De igual manera, se agradece a Patrick Murphy por su aportación en el área de exposición de las viviendas y la clasificación de las mismas en función de su vulnerabilidad.

\section{References}

ABRAHAMSON N.A., SILVA W.J. and KAMAI R., 2013. Update of the AS08 Ground-Motion Prediction Equations Based on the NGA-West2 Data Set. PEER 2013/04.

BOE, 2007. Boletín Oficial del Estado del 29 de julio de 2007 en el que se publica el "REAL DECRETO 1071/2007, de 27 de julio, por el que se regula el sistema geodésico de referencia oficial en España". 27: 35986 - 35989. Avalaible: http://www.boe.es/boe/dias/2007/08/29/pdfs/A35986-35989.pdf

BRUNE J.N., 1968. Seismic moment, seismicity, and rate of slip along major fault zones. Journal of Geophysical Research. 73, pp. 777-784.

CORNELL C.A., 1968. Engineering seismic risk analysis. Bulletin of the Seismological Society of America. 58, pp. 15831606.

CAMPBELL K.W. and BOZORGNIA Y., 2013. NGAWest2 Campbell-Bozorgnia Ground Motion Model for the Horizontal Components of PGA, PGV, and 5\%-Damped Elastic Pseudo- Acceleration Response Spectra for Periods Ranging from 0.01 to 10 s. PEER 2013/06.

CAPOTE, R., DE VICENTE, G. and GONZÁLEZ-CASADO, J.M., 1990. Evolución de las deformaciones alpinas en el Sistema Central Español (S.C.E.). Geogaceta 7, pp. 20-22.

COBURN, A. and SPENCE, R., 2002. Earthquake protection. 2nd edition. Ed. John Wiley \& Sons Ltd, Chichester, UK.

ESTEVA, L., 1968. Bases para la formulación de diseño sísmico (Doctoral dissertation, PhD thesis, Faculty of engineering, UNAM).

FEMA, 2003. HAZUS-MH MR4 Technical Manual. Washington, D.C.

FEMA, 2005. Improvement of Nonlinear Static Seismic Analysis Procedures, FEMA 440, Prepared by Applied Technology Council (ATC-55 Project), Washington, D.C., United States, June 2005.

FEMA, 2005. HAZUS-MH MR4 Technical Manual. Washington, D.C. Federal Emergency Management Agency (2005). Improvement of Nonlinear Static Seismic Analysis Procedures, FEMA 440, Prepared by Applied Technology Council (ATC-55 Project), Washington, D.C., United States, June 2005.

FERNANDES, R. M. S., MIRANDA, J.M., MEIJNINGER, B.M.L., BOS, M.S., NOOMEN, R., BASTOS, L., AMBROSIUS, B.A.C. and RIVA, .E.M., 2007. Surface velocity field of the Ibero-Maghrebian segment of the Eurasia-Nubia plate boundary. Geophysical Journal International 169(1), pp. 315- 324.

GARCÍA-MAYORDOMO J., INSUA-ARÉVALO J., MARTíNEZ-DÍAZ J., PEREA H., ÁLVAREZ-GÓMEZ J.A., MARTÍNGONZÁLEZ F., GONZÁLEZ A., LAFUENTE P., PÉREZ-LÓPEZ R., RODRÍGUEZ-PASCUA M.A., GINER- ROBLES J., AZAÑÓN J.M., MASANA E. and MORENO X., 2010. Modelo integral de zonas sismogénicas de España. Resúmenes de la $1^{\text {a }}$ Reunión Ibérica sobre Fallas Activas y Paleosismología, Sigüenza, España.

GARCÍA-MAYORDOMO J., INSUA-ARÉVALO J.M., MARTÍNEZ- DÍAZ J.J., JIMÉNEZ-DÍAZ A., MARTÍN- BANDA R., MARTÍN- ALFAGEME S., ÁlVAREZ-GÓMEZ J.A., RODRÍGUEZ-PECES M., PÉREZ-LÓPEZ R., RODRÍGUEZPASCUA M.A., MASANA E., PEREA H., MARTÍN-GONZÁLEZ F., GINER-ROBLES J., NEMSER E.S., CABRAL J. and THE QAFI COMPILERS., 2012a. La Base de Datos de Fallas Activas en el Cuaternario de Iberia (QAFI v.2.0). Journal of Iberian Geology, 38(1), pp. 285-302.

GUTENBERG B. and RICHTER C.F., 1944. Frequency of earthquakes in California. Bulletin of the Seismological Society of America. 34, pp. 185-188. 
HAZUS, M.H., 2003. Multi-hazard Loss Estimation Methodology. Technical manual. Federal Emergency Management Agency (FEMA), Washington DC, USA. pp. 712.

IGME, ENRESA, 1998. Mapas Neotectónico y Sismotectónico de España a escala 1:1.000.000. Vol. I (237pp.) y Vol. II, 167 pages.

IGN, INSTITUTO GEOGRÁFICO NACIONAL, 2016. Catálogo de Terremotos. Disponible en: http://www.ign.es/web/ign/portal/sis-catalogo-terremotos.

IGN-UPM, GRUPO DE TRABAJO, 2012. Actualización de Mapas de Peligrosidad Sísmica de España 2012. Editorial Centro Nacional de Información Geográfica, Madrid. ISBN: 978-84-416-2685-0.

MILUTINOVIC, Z.V. and TRENDAFILOSKI, G.S., 2003. An advanced approach to earthquake Risk scenarioswith 87 applications to different European towns. WP4: Vulnerability of current buildings, pp. 110.

MUÑOZ-MARTíN, A., 1997. Evolución geodinámica del borde oriental de la cuenca del Tajo desde el Oligoceno hasta la actualidad. Ph. Thesis, Univ. Complutense de Madrid, 331 pp.

NOCQUET, J.M., 2012. Present-day kinematics of the Mediterranean: A comprehensive overview of GPS results, Tectonophysics 579, pp. 220-242.

PEROUSE, E., VERNANT, P., CHERY, J., REILINGER, R. and MCCLUSKY, S., 2010. Active surface deformation and sub-lithospheric processes in the western Mediterranean constrained by numerical models. Geology 38(9), pp. 823826 Boulder.

RIVAS-MEDINA A., 2014. Contribución metodológica para incorporar fallas activas en la modelización de la fuente dirigida a estimaciones de peligrosidad sísmica. Aplicación al sur de España. Tesis Doctoral. Universidad Politécnica de Madrid.

SEBER, D., BARAZANGI, M., IBENBRAHIM, A. and DEMNATI, A., 1996. Geophysical evidence for lithospheric delamination beneath the Alboran Sea and Rif-Betic mountains. Nature 379, pp. 785-790.

STIRLING M.W., MCVERRY G.H. and BERRYMAN K.R., 2002. A new seismic hazard model of New Zealand. Bulletin of the Seismological Society of America. 92, pp. 1878-1903.

STIRLING, M.W., GERSTENBERGER, M.C., LITCHFIELD, N.J., MCVERRY, G.H., SMITH, W.D., PETTINGA, J. and BARNES, P., 2008. Seismic hazard of the Canterbury region, New Zealand: New earthquake source model and methodology. Bulletin of the New Zealand National Society for Earthquake. Engineering. 41, pp. 51-67.

UNDRO, 1979. Natural Disasters and Vulnerability Analysis, Report of Experts group meeting, Genova, Julio 1979.

WELLS D.L. AND COPPERSMITH K.J., 1994. New empirical relationships among magnitude, rupture length, rupture width, rupture area, and surface displacement. Bulletin of the Seismological Society of America. 84, pp. 974-1002. 